\title{
Adaptive coarse graining, environment, strong decoherence, and quasiclassical realms
}

\author{
Murray Gell-Mann ${ }^{1, *}$ and James B. Hartle ${ }^{1,2, \dagger}$ \\ ${ }^{1}$ Santa Fe Institute, Santa Fe, New Mexico 87501, USA \\ ${ }^{2}$ Department of Physics, University of California, Santa Barbara, Santa Barbara, California 93106-9530, USA
}

(Received 14 January 2014; published 21 May 2014)

\begin{abstract}
Three ideas are introduced that when brought together characterize the realistic quasiclassical realms of our quantum universe as particular kinds of sets of alternative coarse-grained histories defined by quasiclassical variables: (i) branch-dependent adaptive coarse grainings that can be close to maximally refined and can simplify calculation, (ii) narrative coarse grainings that describe how features of the universe change over time and allow the construction of an environment, and (iii) a notion of strong decoherence that characterizes realistic mechanisms of decoherence.
\end{abstract}

DOI: 10.1103/PhysRevA.89.052125

PACS number(s): 03.65.Ta, 03.65.Yz, 98.80.Qc

\section{INTRODUCTION}

A striking feature of our quantum universe is the wide range of time, place, and scale on which the deterministic laws of classical physics hold to an excellent approximation. These realms of classical predictability extend over the whole of the visible universe from just after the big bang to the far future. What is the origin of this classical predictability in a quantum mechanical universe characterized fundamentally by indeterminacy and distributed probabilities? ${ }^{1}$

This paper is one of a series [1-11] aimed at characterizing the realistic quasiclassical realm(s) of our quantum universe as particular kinds of decoherent sets of coarse-grained alternative histories defined by quasiclassical variables. To this end we introduce three (connected) ideas: branch-dependent adaptive coarse grainings, a general notion of a narrative set of alternative histories, and a notion of strong decoherence to characterize realistic mechanisms of decoherence. We have discussed some of these before, ${ }^{2}$ but this discussion we believe is simpler, more general, more connected, and more quantitatively developed. The advantages and importance of these ideas are as follows.

Branch-dependent adaptive coarse grainings. These allow for the possibility of coarse grainings that are close to maximal, as refined as possible, consistent with decoherence and classicality. That way the quasiclassical realms can be a property of our universe and not just our choice. This kind of coarse graining can simplify calculation and act against premature filling of the Hilbert space by not following low probability branches.

Narrative sets of histories. These give a general characterization of a coarse graining whose histories tell a story about

\footnotetext{
*mgm@santafe.edu

†hartle@physics.ucsb.edu

${ }^{1}$ For an elementary review of the origin of the quasiclassical realms of our universe see, e.g., [1].

${ }^{2}$ For instance, branch dependence was discussed in [2]. Adaptive coarse graining and narratives were discussed qualitatively in [11]. What is added here is the explicit formulation of these ideas and the connection with decoherence. A notion of strong decoherence was introduced in [10]. The formulation given here is less abstract, more accessible, and connects with other ideas such as an environment.
}

how features of the universe change over time. They also allow the construction of an environment. They therefore put the notion of environment in its proper place as a consequence of a narrative coarse graining and not as a separate postulate of quantum mechanics.

Strong decoherence. This is a more realistic, but still general, notion of decoherence that characterizes realistic mechanisms of decoherence where records are created in variables other than those followed. The orthogonality of these records produces decoherence. Strong decoherence ensures that the past remains permanent as a set of histories is fine grained by extending it into the future.

We work in the decoherent (or consistent) histories formulation (DH) of the quantum mechanics of a closed system, most generally the universe. ${ }^{3}$ DH contains within it other formulations of quantum theory as approximations appropriate for particular circumstances. These include the Copenhagen formulation for measurement situations and the open system formulations based on an assumed environment. ${ }^{4}$

The predictions of DH for laboratory experiment will therefore generally not differ significantly from those of the Copenhagen formulation when the conditions for that approximation apply. ${ }^{5}$ Rather DH is a more general formulation of quantum theory suitable, for example, for describing the orbit of the Moon when it is not being observed, density fluctuations in the early universe when there were neither observers or observations, and the quasiclassical realms of our universe, which are the focus of this paper. DH will be briefly reviewed in Sec. II.

Some of the results of this paper help explain how the approximations mentioned above emerge from $\mathrm{DH}$. The coarse graining necessary for the decoherence of histories naturally specifies a system-environment split of the Hilbert space for narrative sets of alternative histories. Strong decoherence leads naturally to a density matrix defined on the system Hilbert space.

The paper is structured as follows. Section II reviews decoherent histories quantum mechanics, largely to establish our notation. Branch-dependent coarse grainings and adaptive

\footnotetext{
${ }^{3}$ See $[7,12,13]$ for classic expositions and [14] for a tutorial.

${ }^{4}$ See, e.g., [15] for a review.

${ }^{5}$ See, e.g., [4], Secs. II.10 and II.6.4, and [16].
} 
coarse grainings are described in Sec. III. The notion of a narrative framework is introduced in Sec. IV and the systemenvironment split that follows from it in Sec. V. Section VI gives a simplified account of strong decoherence and Sec. VII is concerned with the notions of records and density matrices that follow from it. There are brief conclusions in Sec. VIII.

\section{HISTORIES, COARSE GRAININGS, AND DECOHERENCE}

Largely to establish notation, we give a very brief account of some essential elements of the modern synthesis of ideas characterizing the quantum mechanics of closed systems that we call decoherent histories quantum mechanics.

\section{A. A model closed quantum system}

We consider a closed quantum system, most generally the universe, in the approximation that gross quantum fluctuations in the geometry of space-time can be neglected. ${ }^{6}$ The closed system can then be thought of as a large (say $\gtrsim$ $20000 \mathrm{Mpc}$ ), perhaps expanding box of particles and fields in a fixed background space-time. Everything is contained within the box, galaxies, planets, observers and observed, measured subsystems, any apparatus that measures them, and, in particular, any human observers including us. This is a manageable model of the most general physical context for prediction.

There is a Hilbert space $\mathcal{H}$ for the contents of the box. The essential theoretical inputs to the process of prediction are the Hamiltonian $H$ governing evolution and the quantum state of the universe, which we assume to be a $\operatorname{pure}^{7}|\Psi\rangle$.

\section{B. Histories}

We will work in the Schrödinger picture where one operator represents the same quantity at all times. Operators in the Schrödinger picture will be distinguished from Heisenberg picture operators by carets (hats), viz., $\hat{\mathcal{O}}$.

Sets of yes or no alternatives at one moment of time $t$ are represented by an exhaustive set of orthogonal projection operators $\left\{\hat{P}_{\alpha}\right\}, \alpha=1,2,3, \ldots$, satisfying

$$
\sum_{\alpha} \hat{P}_{\alpha}=I, \quad \hat{P}_{\alpha} \hat{P}_{\beta}=\delta_{\alpha \beta} \hat{P}_{\alpha} .
$$

These conditions ensure that the projections represent an exhaustive set of exclusive alternatives. A completely finegrained description of a quantum system at a moment of time is provided by a set of one-dimensional projections. All other sets are coarse grained.

The most general objective of quantum theory is the prediction of the probabilities of individual members of sets of alternative coarse-grained time histories of the closed system. For instance, we might be interested in alternative histories of

\footnotetext{
${ }^{6}$ For the generalizations that are needed for quantum space-time see, e.g., $[17,18]$.

${ }^{7}$ From the perspective of the time-neutral formulation of quantum theory (see, e.g., [6]), we are assuming also a final condition of ignorance.
}

the center of mass of the Earth in its progress around the Sun or in histories of the correlation between the registrations of a measuring apparatus and a measured property of a subsystem. Histories are essential for defining classical behavior. For example, we say that the Earth moves in a classical orbit when the probability from $H$ and $\Psi$ is high for a history of its motion that is correlated in time by Newton's laws.

An important kind of set of alternative histories is specified by sets of alternatives at a sequence of times $t_{1}<t_{2}<\cdots<t_{n}$. An individual history $\alpha$ in such a set is a particular sequence of alternatives $\alpha \equiv\left(\alpha_{1}, \alpha_{2}, \ldots, \alpha_{n}\right)$ at the times ${ }^{8} t_{1}<t_{2}<$ $\cdots<t_{n}$. Such a set of histories has a branching structure in which a history up to any given time $t_{m} \leqslant t_{n}$ branches into further alternatives at later times. Each history is a branch of the branching structure.

Individual histories are represented by the chains $\hat{C}_{\alpha}$ of the projection operators that define the alternatives $\alpha=$ $\left(\alpha_{1}, \alpha_{2}, \ldots, \alpha_{n}\right)$ at the times $t_{1}<t_{2}<\cdots<t_{n}$ with unitary evolution between times specified by the Hamiltonian. The simplest examples are sets of histories defined by the same sets alternatives $\left\{\hat{P}_{\alpha}\right\}$ at the series of times. These histories are represented by the operators

$$
\begin{aligned}
\hat{C}_{\alpha} \equiv & \hat{P}_{\alpha_{n}} e^{-i H\left(t_{n}-t_{n-1}\right) / \hbar} \hat{P}_{\alpha_{n-1}} e^{-i H\left(t_{n-1}-t_{n-2}\right) / \hbar} \\
& \times \cdots \hat{P}_{\alpha_{1}} e^{-i H\left(t_{1}-t_{0}\right) / \hbar}
\end{aligned}
$$

where $t_{0}<t_{1}$ is an initial time where the initial state of the box $\hat{\Psi}\left(t_{0}\right)$ is specified.

For any individual history $\alpha$, its branch state vector at time $t_{n}$ is defined by

$$
\hat{\Psi}_{\alpha}\left(t_{n}\right)=\hat{C}_{\alpha} \hat{\Psi}\left(t_{0}\right)
$$

The branch state vector $\hat{\Psi}_{\alpha}(t)$ can be defined at any other time by the unitary evolution of (2.3) with $H$. Evidently, from (2.1)

$$
\hat{\Psi}(t)=\sum_{\alpha} \hat{\Psi}_{\alpha}(t) .
$$

\section{Decoherence}

When probabilities can be consistently assigned to the individual histories in a set, they are given by

$$
p(\alpha)=\left\|\hat{\Psi}_{\alpha}(t)\right\|^{2}=\left\|\hat{C}_{\alpha} \hat{\Psi}\left(t_{0}\right)\right\|^{2} .
$$

However, because of quantum interference, probabilities cannot be consistently assigned to every set of alternative histories that may be described. Negligible interference between the branches of a set

$$
\left(\hat{\Psi}_{\alpha}(t), \hat{\Psi}_{\beta}(t)\right) \approx 0, \quad \alpha \neq \beta,
$$

is a sufficient condition for the probabilities (2.5) to be consistent with the rules of probability theory. The orthogonality of the branches is approximate in realistic situations. However, we mean by (2.6) equality to an accuracy that defines probabilities well beyond the standard to which they can be checked or the physical situation modeled. Sets of histories for which the interference is negligible according to (2.6) are said

\footnotetext{
${ }^{8}$ More generally, alternatives can be extended over time [19].
} 
to (medium) decohere. ${ }^{9}$ Medium decoherence is the weakest of known conditions that are consistent with elementary notions of the independence of isolated systems [20].

For characterizing quasiclassical realms stronger notions of decoherence characterizing realistic mechanisms of decoherence can be useful [10]. A wide class of stronger notions replaces (2.6) with

$$
\left(\hat{\Psi}_{\alpha}(t), \mathcal{O} \hat{\Psi}_{\beta}(t)\right) \approx 0, \quad \alpha \neq \beta,
$$

for some class of operators $\mathcal{O}$ including the identity. The notion of strong decoherence that we introduced in [10] is an example that we will discuss in Sec. VI.

\section{Quasiclassical variables}

Quasiclassical realms are defined by coarse grainings based on quasiclassical variables. These are averages over small volumes of densities of approximately conserved quantities such as energy, momentum, and numbers, such as baryon number, in epochs when they are conserved. The approximate conservation of these quantities is the source of their classical predictability in the face of the noise that typical mechanisms of decoherence produce (see, e.g., [1,2,5,11,21,22]).

A quasiclassical coarse graining is specified by three things: (1) a sequence of time steps $t_{1}, t_{2}, \ldots, t_{n}$, (2) a partition of space into volumes $V(\vec{y})$ labeled by a triple of integers $\vec{y}$, and (3) an exhaustive set of exclusive ranges of coarse grained values $\left\{\Delta_{\beta}\right\}$ for the averages over each volume $\vec{y}$ of each of the quantities energy density, momentum density, and number density, at each time step. For example, if $\epsilon(\vec{x})$ is the energy density the average $\bar{\epsilon}(\vec{x})$ is

$$
\bar{\epsilon}(\vec{y}) \equiv \frac{1}{V(\vec{y})} \int_{V(\vec{y})} d^{3} x \in(\vec{x}),
$$

with similar expressions for the other quantities.

A particular history is represented by sequences of particular ranges $\Delta_{\beta}$ at each time step, for each volume, for each variable. For more detail on the construction and its consequences see [11].

As described here, quasiclassical coarse grainings are conceptually simple but notationally messy. We will therefore use a highly condensed notation for them. We denote by $\hat{P}_{\alpha_{k}}^{k}$ the projections (or products of nearby projections) at each time step. The superscript $k$ denotes the time step and the coarse graining at that time step. That is, it stands for the time step and the coarse-graining ingredients (2) and (3) mentioned above at that time step. The index $\alpha_{k}$ denotes the particular alternative at the time step $k$. That is, it stands for the particular ranges $\Delta_{\beta}$, for each quasiclassical variable, in each volume $\vec{y}$.

\section{BRANCH-DEPENDENT COARSE GRAININGS}

This section develops the idea of branch-dependent coarse grainings in which the set of alternatives defining the branches

\footnotetext{
${ }^{9}$ Thus, by decoherence we do not mean the approach to diagonality over time of some density matrix. Equation (2.6) is a condition on histories. However, realistic mechanisms of decoherence can lead to appropriately defined density matrices becoming diagonal as described in Sec. VII C of this paper and in Sec. II.6.4 of [4].
}

at one time depends on the specific history (branch) that preceded it. The idea of adaptive branch-dependent coarse grainings is also introduced in which coarse grainings are adapted to changing physical situations. Sets of histories that describe realistic physical situations in the universe are almost always branch dependent. Adaptive coarse grainings are the efficient way of exhibiting the interesting features of these situations. We begin with two illustrative examples, the first on the scale of the laboratory and the second on the scale of the cosmos.

\section{A. Examples of branch dependence}

\section{A model measurement situation}

Imagine a closed system consisting of an isolated laboratory containing an experimenter. The laboratory is equipped with apparatus for measuring the spin of an isolated electron in a prepared state. The apparatus can be adjusted to measure the spin along any direction the experimenter chooses. The experimenter, the apparatus, and the electron are all quantum mechanical physical systems within the closed laboratory. At time $t_{1}$ the experimenter flips a coin (or uses a quantum random bit generator) to decide whether to measure the spin along the $x$ axis or $z$ axis: Heads it is the $z$ direction; tails it is the $x$ direction. At time $t_{2}$ the experimenter carries out the measurement. The relevant histories for describing this situation consist of chains of projections at two times. The alternatives at the first time describe the direction chosen for the spin measurement, $\left(\hat{P}_{\text {meas } x}, \hat{P}_{\text {meas } z}\right)$ in what we hope is an obvious notation. At the second time the alternatives are $\left(\hat{P}_{+}^{z}, P_{-}^{z}\right)$ if the first alternative was $\hat{P}_{\text {meas } z}$ and $\left(\hat{P}_{+}^{x}, \hat{P}_{-}^{x}\right)$ if the first alternative was $\hat{P}_{\text {meas } x}$, where $(+,-)$ denote the values of the spin projections along the direction chosen. That is branch dependence. The relevant set of histories is thus

$$
\hat{P}_{+}^{z} U \hat{P}_{\text {meas } z}, \quad \hat{P}_{-}^{z} U \hat{P}_{\text {meas } z}, \quad \hat{P}_{+}^{x} U \hat{P}_{\text {meas } x}, \quad \hat{P}_{-}^{x} U \hat{P}_{\text {meas } x},
$$

where $U \equiv \exp \left[-i H\left(t_{2}-t_{1}\right) / \hbar\right]$. Thus branch-dependent histories are needed to describe the simplest measurement when system, apparatus, and observer are all treated as subsystems of one closed system.

The set of histories (3.1) describes all the alternatives that can happen in this limited measurement model. One could say that it is a third-person description of the possible measured histories (e.g., as in [23]). However, suppose that the result of the experimenter's coin flip at $t_{1}$ is heads so that the spin in the $z$ direction will be measured at time $t_{2}$. The experimenter may be interested only in histories that describe the outcome of the projected experiment from the first-person point of view and not the outcomes of the experiment that could have been carried out if the coin had come up tails. The experimenter would then use the set of histories

$$
\hat{P}_{+}^{z} U \hat{P}_{\text {meas } z}, \quad \hat{P}_{-}^{z} U \hat{P}_{\text {meas } z}, \quad U \hat{P}_{\text {meas } x}
$$

(leaving out zero branches). This is coarser grained than (3.1) but still branch dependent. Either way, branch-dependent sets of histories are needed to describe realistic measurement situations when system, apparatus, and experimenter are all treated as subsystems of one closed system. 


\section{Planet formation}

Quasiclassical realms have to be branch dependent in order to have a chance of being maximally refined with respect to decoherence and classicality (and therefore of being a feature of the universe and not our choice). For example, consider the formation of the Earth, starting with a protostellar cloud. A relatively coarse-grained description of the gas might be appropriate in the protostellar cloud, to be followed by increasingly finer-grained descriptions at the locations where a star (the Sun) condensed, where a planet (the Earth) at 1AU won the battle of accretion in the circumstellar disk, etc. The higher density in the condensed region means that collision rates will be higher, so the mechanisms of decoherence will operate more quickly. It also means that the same inertia is achieved in smaller volumes. This means that the volumes of the quasiclassical realm can be smaller and the times between alternatives can be shorter and still exhibit classical predictability in the face of the quantum noise produced by the mechanisms of decoherence. The more refined set with smaller volumes and shorter times is closer to maximality.

The locations where the Sun condensed or the Earth formed will be different on different branches. Indeed, there will be branches where they did not condense at all. The coarse grainings described above are therefore branch dependent.

\section{B. Branch dependence in general}

We now discuss branch dependence in general. It will be convenient in this Schrödinger picture to consider histories on a fixed time interval starting with $t_{0}$ and ending at $T$. (This is no restriction at all since the end points are arbitrary.) We can then consider histories defined at a variable number $n$ of time steps within this interval.

In a branch-dependent coarse graining the quasiclassical yes or no alternatives at a given time are represented by an exhaustive set of exclusive projection operators satisfying (2.6). We denote those at time $t_{k}$ by ${ }^{10}$

$$
\hat{P}_{\alpha_{k}}^{k \alpha_{k-1} \cdots \alpha_{1}} \text {. }
$$

The upper indices label the set. The quantity $k$ labels both the time step and the coarse graining used at that time, as discussed in Sec. II D. For example, we might employ projections on a certain exhaustive set of exclusive ranges of quasiclassical variables defined by one set of averaging volumes $V(\vec{y})$ at one time [see (2.8)] and use quasiclassical variables defined by different volumes at a different time or different ranges $\Delta_{\beta}$ at different times, etc. The upper indices $\left(\alpha_{k-1}, \ldots, \alpha_{1}\right)$ indicate branch dependence: The set of alternatives at time step $k$ depends on the previous alternatives defining a particular history as in the examples given above. Allowing only dependence on past alternatives means that causality is built in at a basic level. The subscript $\alpha_{k}$ denotes

\footnotetext{
${ }^{10}$ Unfortunately, in previous papers we arranged the indices differently. For instance, in [11] we wrote $P_{\alpha_{k}}^{k}\left(t_{k} ; \alpha_{k-1}, \ldots, \alpha_{1}\right)$ for Heisenberg picture projections and in [10] we wrote all the indices downstairs except $k$. These all mean the same thing. The notation used here cleanly separates the description of the set (upper indices) from the particular alternative within the set (lower index).
}

the particular alternative in the set, a particular range of the quasiclassical variables in all the volumes.

The times and the number of time steps are also branch dependent. That must be the case if we aim at sets that are maximally refined consistent with decoherence and classicality. For instance, sets of projections can be closer together in time when they refer to regions where decoherence is more rapid than elsewhere, as in the planet formation example above. Thus we should write

$$
t_{k}=t_{k}\left(\alpha_{k-1}, \ldots, \alpha_{1}\right)
$$

and a similar formula for the total number of steps $n$ in the range $t_{0}<t<T$. However, in order not to expand an already complex notation, in this paper we will consider a fixed sequence of times $t_{1}, \ldots, t_{n}$ that is refined enough to accommodate all branches and use a trivial set of alternatives $(0, I)$ on particular branches where there needs to be more time separation between nontrivial alternatives.

Histories are then represented by class operators incorporating the projections interrupted by unitary evolution. For example, with just three intermediate times we have ${ }^{11}$

$$
\begin{aligned}
\hat{C}_{\alpha_{3} \alpha_{2} \alpha_{1}}^{3}= & \hat{P}_{\alpha_{3}}^{3 \alpha_{2} \alpha_{1}} e^{-i H\left(t_{3}-t_{2}\right) / \hbar} \hat{P}_{\alpha_{2}}^{2 \alpha_{1}} e^{-i H\left(t_{2}-t_{1}\right) / \hbar} \\
& \times \hat{P}_{\alpha_{1}}^{1} e^{-i H\left(t_{1}-t_{0}\right) / \hbar} .
\end{aligned}
$$

The formulas for longer sequences of times (or fewer) should be evident.

\section{Adaptive branch-dependent coarse grainings}

Adaptive coarse grainings are branch dependent in a rule based way. For example, we may adapt the coarse graining to follow the motion of the Earth, or what happens on its surface, by choosing alternatives at a future time that describe what goes on at its future location and ignore what happens at other locations. Appropriately adaptive coarse grainings can reduce the proliferation of branches and simplify the calculation of decoherence by focusing on histories of interest and ignoring others. A simple example of a general adaptive rule is, at any one time step, not to further refine branches that already have negligible probabilities. Further division of such branches can only reduce the probabilities [24]. A wave packet moving in one dimension provides a very simple example. The motion of the packet can be followed with an adaptive coarse graining that fine grains only near the center of the wave packet as it moves through successive time steps.

\section{Medium decoherence of branch-dependent sets of histories}

Assume that the universe has a pure initial state at time $t_{0}$, which we denote by $\hat{\Psi}\left(t_{0}\right)$ in the Schrödinger picture. We will also use $\hat{\Psi}^{0} \equiv \hat{\Psi}\left(t_{0}\right)$ as an alternate notation consistent with the conventions for the projections in (3.3). Consider a set of alternative histories defined by sequences of projections of the form (3.3) at a sequence of times $t_{1}, \ldots, t_{n}$ and represented

\footnotetext{
${ }^{11}$ Since the indices on the $\hat{P}$ 's sometimes represent a set and sometimes label an alternative, a convention has to be chosen for how they are placed on the $\hat{C}$ 's. This one in (3.5) is picked for later convenience.
} 
by class operators (3.5). As above, to keep the notation manageable we will consider histories with just three times $t_{1}, t_{2}, t_{3}$. The branch state vectors for individual histories are [cf. (2.3)]

$$
\hat{\Psi}_{\alpha_{3}}^{3 \alpha_{2} \alpha_{1}} \equiv \hat{C}_{\alpha_{3} \alpha_{2} \alpha_{1}} \hat{\Psi}^{0}
$$

The sum of the branches gives back the state at time step 3 , that is,

$$
\hat{\Psi}^{3}=\sum_{\alpha_{3} \alpha_{2} \alpha_{1}} \hat{\Psi}_{\alpha_{3}}^{3 \alpha_{2} \alpha_{1}},
$$

as is easily verified from (3.5) and (2.1).

These Schrödinger picture branch state vectors can be evolved to any time step with the Hamiltonian, e.g.,

$$
\hat{\Psi}_{\alpha_{3}}^{4 \alpha_{2} \alpha_{1}} \equiv e^{-i H\left(t_{4}-t_{3}\right) / \hbar} \hat{C}_{\alpha_{3} \alpha_{2} \alpha_{1}}^{3} \hat{\Psi}^{0} .
$$

Medium decoherence is the requirement that all the branches be mutually orthogonal:

$$
\left(\hat{\Psi}_{\alpha_{3}^{\prime}}^{3 \alpha_{2}^{\prime} \alpha_{1}^{\prime}}, \hat{\Psi}_{\alpha_{3}}^{3 \alpha_{2} \alpha_{1}}\right)=\delta_{\alpha_{3}^{\prime} \alpha_{3}} \delta^{\alpha_{2}^{\prime} \alpha_{2}} \delta^{\alpha_{1}^{\prime} \alpha_{1}} p\left(\alpha_{3} \alpha_{2} \alpha_{1}\right)
$$

where $p\left(\alpha_{3} \alpha_{2} \alpha_{1}\right)$ are the probabilities of the histories.

\section{NARRATIVE REALMS AND COMMON FRAMEWORKS}

\section{A. Narrative realms}

Narrative realms tell a story through their probabilities about how features of the universe change in time. Often these stories concern the evolution in a quasiclassical realm of unique, identifiable objects: ${ }^{12}$ the galaxy NGC4258, the planet Mars, the Andes, eddies in your bathtub, individual human beings, and so forth. For a realm to be a narrative whose probabilities describe the history of an object the sets of projections $\left\{\hat{P}_{\alpha_{k}}^{k \alpha_{k-1} \cdots \alpha_{1}}\right\}$ must be chosen to that end. At a minimum they must follow the object at different times.

It will be useful to define narrative coarse grainings more generally than just those pertaining to objects. In general, we need to capture precisely the notion that a narrative coarse graining follows similar variables at a series of times.

The simplest example of a rule generating a narrative coarse graining is to use the same set of alternatives at all times. That is, the narrative is given by histories that have the same set of Schrödinger picture projections $\left\{\hat{P}_{\alpha}\right\}$ for all time steps. However, this simple rule does not allow for branch dependence.

A rule more general than identity that captures the notion of similar variables at different times is to require that the Schrödinger picture projections commute, ${ }^{13}$

\footnotetext{
${ }^{12}$ We assume that our box is small enough that unique objects can exist in the sense that the probability for their replication elsewhere is negligible. We thus are not considering the vast universes of contemporary inflationary theory in which everything is duplicated someplace else. For what to do then see [23].

${ }^{13}$ As discussed in Sec. II D for quasiclassical variables that do not commute either we would have to find that they effectively commute as in [21,22] or divide $k$ up into nearby separated time steps. We will not complicate an already extended notation to indicate this.
}

viz.,

$$
\left[\hat{P}_{\alpha_{k}}^{k \alpha_{k-1} \cdots \alpha_{1}}, \hat{P}_{\alpha_{k}^{\prime}}^{k^{\prime} \alpha_{k^{\prime}-1}^{\prime} \cdots \alpha_{1}^{\prime}}\right]=0 .
$$

Evidently identical projections at different times commute, but the condition (4.1) is much more general and consistent with branch dependence. We will call this the narrative condition.

\section{B. Common frameworks}

The narrative condition (4.1) immediately leads to the notion of a common framework for narrative histories. There is a basis in $\mathcal{H}$ in which all the $\hat{P}$ satisfying the narrative condition (4.1) are simultaneously diagonal. That means that there is an exhaustive set of mutually exclusive projections $\left\{\hat{P}_{\gamma}\right\}$ that is an operator basis for all the $\hat{P}$ in the histories. Specifically

$$
\hat{P}_{\alpha_{k}}^{k \alpha_{k-1} \cdots \alpha_{1}}=\sum_{\gamma \in\left(k \alpha_{k} \cdots \alpha_{1}\right)} \hat{P}_{\gamma},
$$

where the notation means that the $P$ of the histories project onto orthogonal subspaces of $\mathcal{H}$ that are unions of the subspaces of the common framework.

For quasiclassical realms one can think of the $\hat{P}_{\gamma}$ as defined by alternative values of quasiclassical variables using a partition of space into very small volumes for averaging approximately conserved quantities, the same partition at all times. There is no requirement that histories of these $\hat{P}_{\gamma}$ be decoherent. The coarse grainings for branch-dependent sets of histories are defined by grouping these small volumes into appropriate larger ones in a branch-dependent way that does define a decoherent set of histories.

\section{ENVIRONMENTS}

\section{A. Use of environments}

There is a long and important history of analyzing decoherence phenomena in terms of the interaction between a subsystem and an environment. Seminal papers in the modern quantum mechanics of closed systems include those of Fano [25], Joos and Zeh [26], and the many of Zurek and co-workers, reviewed, for example, in [15]. Important earlier work, on which the present discussion relies, includes the papers of Feynman and Vernon [27], Caldeira and Leggett [28], and our own [5]. In these treatments one set of fundamental coordinates (say, $x$ ) define the subsystem, the rest (say, $Q$ ) define the environment. For instance, the subsystem might be a single dust particle interacting with photons of the cosmic background radiation radiation constituting an environment. Corresponding to this division of coordinates there is a tensor product factoring of the Hilbert space $\mathcal{H}$,

$$
\mathcal{H}=\mathcal{H}^{s} \otimes \mathcal{H}^{e}
$$

with $\mathcal{H}^{s}$ spanned by center-of-mass position of the dust grain and $\mathcal{H}^{e}$ by the field variables of the photons and the internal relative coordinates of the atoms in the grain. The Hilbert space $\mathcal{H}^{s}$ is for the system and $\mathcal{H}^{e}$ defines its environment.

The ubiquity of models assuming a system-environment split has given some the impression that such a split must be postulated to formulate the quantum mechanics of closed systems. This is not correct. 
When quantum mechanics is formulated generally there is no fundamental system-environment split. The general notion is rather coarse graining. As mentioned above, the most general objective of quantum theory is the prediction of the probabilities of sets of alternative coarse-grained histories of a closed system. Coarse graining is inevitable because there are no nontrivial fine-grained sets of histories that decohere. It is the coarse graining that specifies what is followed and what is ignored. No additional separate postulate of a system-environment split is needed to extract predictions from the theory. Whatever notion of system and environment may be available follows from the coarse graining defining a particular set of alternative histories and will differ from one set of alternative histories to another and indeed from one time to another. Environments are not postulated; they are constructed from sets of sets of histories. We now describe how that works.

\section{B. Constructing environments}

First, consider branch-independent realms. We will return to the more general branch-dependent case immediately afterward.

For a given branch-independent realm a systemenvironment split is defined at one time when the Hilbert space $\mathcal{H}$ is a tensor product as in (5.1) and all the projections defining the histories are of the form

$$
\hat{P}_{\alpha}=\hat{P}_{\alpha}^{s} \otimes I^{e}
$$

at that time for that realm.

The important mathematical result is the converse. Given a set of commuting projection operators $\left\{\hat{P}_{\alpha}\right\}, \alpha=1,2, \ldots$, all defining infinite-dimensional subspaces of $\mathcal{H}$, it is possible to factor the $\mathcal{H}$ as in (5.1) so that all the projections act on one factor as in (5.2). The argument is a simple one. ${ }^{14}$ Since all the projections commute they can be written in a common basis $\{|i\rangle\}$ in the form

$$
\hat{P}_{\alpha}=\sum_{i \in \alpha}|i\rangle\langle i| .
$$

Then it is is just a matter of relabeling the basis $|i\rangle \equiv|\alpha, A\rangle$ to define a tensor product (5.1) on which the projections act as in (5.2).

In the simplest case its is possible to arrange for the projections on the system space to be one dimensional

$$
\hat{P}_{\alpha}^{s} \equiv|\alpha\rangle^{s s}\langle\alpha| \text {. }
$$

The system Hilbert space $\mathcal{H}^{s}$ is spanned by all the $|\alpha\rangle^{s}$ and the environment Hilbert space $\mathcal{H}^{e}$ is spanned by all the $|A\rangle^{e}$. The environment Hilbert space is then as large as possible, allowing the most Hilbert space in which the phases between histories can get lost. That is the best possibility for decoherence.

However, it can be convenient to allocate a little more of the Hilbert space to the system by assigning some of the $|A\rangle^{e}$ to $\mathcal{H}^{s}$. Then the system Hilbert space $\mathcal{H}^{s}$ is spanned by vectors

\footnotetext{
${ }^{14}$ See Appendix A of [29]. There is an obstruction to factorization in the finite-dimensional case arising from the relation between dimensions following from (5.2), specifically $\operatorname{dim}\left(P_{\alpha}\right)=\operatorname{dim}\left(P_{\alpha}^{s}\right) \operatorname{dim}\left(I^{e}\right)$.
}

$|\alpha, r\rangle^{s}$ and

$$
\hat{P}_{\alpha}^{s}=\Sigma_{r}|\alpha, r\rangle^{s s}\langle r, \alpha| .
$$

Thus, in a set of histories defined by sequences of sets of branch-independent commuting projections $\left\{\hat{P}_{\alpha_{k}}^{k}\right\}$, there would be a system-environment split defined at each moment of time, although generally a different split from one moment to the next. For different realms defined by different sets of projections there would be different splits.

\section{System-environment splits for branch-dependent coarse grainings}

In the more realistic branch-dependent case we do not generally have one set of commuting projections at each time. The branch-dependent projections (3.3) need not commute for different values of $\alpha_{n-1}, \ldots, \alpha_{1}$. The simple measurement situation described in Sec. III A 1 is an example. For a general set of branch-dependent histories there will be no notion of environment available even at one time.

A system-environment split of the Hilbert space can be constructed at one time when there is something in common that is followed by all the projections $\left\{\hat{P}_{\alpha_{k}}^{k \alpha_{k-1} \cdots \alpha_{1}}\right\}$. That can constitute the system and the rest is the environment. Mathematically this idea is implemented when all the projections at a given time commute, viz.,

$$
\left[\hat{P}_{\alpha_{k}}^{k \alpha_{k-1} \cdots \alpha_{1}}, \hat{P}_{\alpha_{k}^{\prime}}^{k \alpha_{k-1}^{\prime} \cdots \alpha_{1}^{\prime}}\right]=0 .
$$

This is of the same form as the narrative condition (4.1) but enforced only when the times are the same. As in that discussion, there is now an operator basis for all the $\hat{P}$ and we can write for each time step $k$

$$
\hat{P}_{\alpha_{k}}^{k \alpha_{k-1} \cdots \alpha_{1}}=\sum_{\gamma \in\left(\alpha_{k} \cdots \alpha_{1}\right)} \hat{P}_{\gamma}^{k},
$$

where the sum is over all $\hat{P}_{\gamma}^{k}$ contained in the projection $\hat{P}_{\alpha_{k}}^{k \alpha_{k-1} \cdots \alpha_{1}}$. The common framework $\hat{P}_{\gamma}^{k}$ can then be used to factor the Hilbert space as in Sec. V B and define a systemenvironment split at each time. If the common frameworks $\left\{\hat{P}_{\gamma}^{k}\right\}$ are the same for all times (all $k$ ) then an environment can be defined that is fixed for all time. Sets of histories constructed from the $\hat{P}_{\gamma}^{k}$ themselves are not necessarily decoherent. Rather they provide a common framework for the branch-dependent sets that do decohere.

When a branch-dependent set of histories is a narrative so that (4.1) is satisfied, then there is a common framework for all times (5.7) and one system-environment split for all times. That will be the case for quasiclassical realms since we define them to be narratives.

\section{Constructing common frameworks}

For a given time step $k$ the $\hat{P}_{\gamma}^{k}$ can be constructed from the projections $\hat{P}_{\alpha_{k}}^{k \alpha_{k-1} \cdots \alpha_{1}}$ when these all commute with one another as in (5.6). To see this consider for simplicity $k=2$ and define the operator products

$$
\stackrel{\circ}{P}_{\alpha_{2} \alpha_{2}^{\prime}}^{2 \alpha_{1} \alpha_{1}^{\prime}} \equiv \hat{P}_{\alpha_{2}}^{2 \alpha_{1}} \hat{P}_{\alpha_{2}^{\prime}}^{2 \alpha_{1}^{\prime}}
$$


Since the the $\hat{P}_{\alpha_{2}}^{2 \alpha_{1}}$ commute for with each other for different indices by assumption (5.6), the $\stackrel{P}{P}$ are themselves projectors and the set of them an exhaustive set of exclusive projections. In fact, $\stackrel{\circ}{P}_{\alpha_{2} \alpha_{2}^{\prime}}^{2 \alpha_{1} \alpha_{1}^{\prime}}$ projects on the intersection of the subspaces defined by its constituent projections. The $\hat{P}$ can be recovered from the $\stackrel{\circ}{P}$ by, e.g.,

$$
\hat{P}_{\alpha_{2}}^{2 \alpha_{1}}=\sum_{\alpha_{2}^{\prime} \alpha_{1}^{\prime}} \stackrel{\circ}{P}_{\alpha_{2} \alpha_{2}^{\prime}}^{2 \alpha_{1} \alpha_{1}^{\prime}} .
$$

Thus, the $\hat{P}_{\gamma}^{2}$ in (5.7) are the ${\stackrel{P}{\alpha_{2} \alpha_{2}^{\prime}}}_{2 \alpha_{1} \alpha_{1}^{\prime}}$. The index $\gamma$ ranges over the intersections of the $\hat{P}_{\alpha_{2}}^{2 \alpha_{1}}$.

This explicit construction becomes increasingly complex for later times because all possible products of projections defining the histories enter. The idea is the same; the equations become lengthy.

We can therefore have a basis in $\mathcal{H}$ of the form $|\gamma, B\rangle$, where $\gamma$ labels the intersections and $B$ labels the vectors in the intersections. We can then invoke the arguments in Sec. V B to make a system-environment split where the system Hilbert space $\mathcal{H}^{s}$ is spanned by vectors $|\gamma\rangle$ and the environment Hilbert space by the $|B\rangle$. We now use these ideas to define strong decoherence.

\section{STRONG DECOHERENCE SIMPLIFIED}

As mentioned in the Introduction, a wide class of realistic mechanisms of decoherence is characterized by the creation of orthogonal records leading to a notion of decoherence, which we have called strong decoherence $[10,30]$. The construction of environments for each time step from a common framework in Sec. V permits a simplified but general discussion of strong decoherence. We present that in this section. For simplicity we give the exposition for histories with just three time steps, but the generalization of the formulas to more (or fewer) steps should be evident.

The assumption that all the $\hat{P}$ at a given time commute (5.6) allows a system-environment split at each time as discussed in Sec. V. At time step 3 we would have

$$
\mathcal{H}=\mathcal{H}^{3 s} \otimes \mathcal{H}^{3 e},
$$

where the $\hat{P}$ operate only on the system Hilbert space $\mathcal{H}^{3 s}$ at time step 3. Explicitly this means

$$
\hat{P}_{\alpha_{3}}^{3 \alpha_{2} \alpha_{1}}=\sum_{r_{3}} v_{\alpha_{3} r_{3}}^{3 \alpha_{2} \alpha_{1}} v_{\alpha_{3} r_{3}}^{\dagger 3 \alpha_{2} \alpha_{1}} \otimes I^{3 e}
$$

where the $v$ are a set of orthogonal basis vectors in $\mathcal{H}^{3 s}$ that can be arranged to satisfy

$$
\left(v_{\alpha_{3} r_{3}}^{3 \alpha_{2} \alpha_{1}}, v_{\alpha_{3}^{\prime} r_{3}^{\prime}}^{3 \alpha_{2} \alpha_{1}}\right)^{3 s}=\delta_{\alpha_{3}^{\prime} \alpha_{3}} \delta_{r_{3}^{\prime} r_{3}} .
$$

Since the $\hat{P}$ are an exhaustive set of projections, the set of $v$ for all projections in the set (all $\alpha_{3}$ ) will be a basis for $\mathcal{H}^{3 s}$ and we can expand $\hat{\Psi}_{\alpha_{3}}^{3 \alpha_{2} \alpha_{1}}$ in terms of them, viz.,

$$
\hat{\Psi}_{\alpha_{3}}^{3 \alpha_{2} \alpha_{1}}=\sum_{r_{3}} v_{\alpha_{3} r_{3}}^{3 \alpha_{2} \alpha_{1}} \otimes z_{\alpha_{3} r_{3}}^{3 \alpha_{2} \alpha_{1}} .
$$

The coefficients $z_{\alpha_{3} r_{3}}^{3 \alpha_{2} \alpha_{1}}$ are vectors in the environment Hilbert space at time step $3, \mathcal{H}^{3 e}$.
The condition for medium decoherence (3.9) then becomes

$$
\begin{aligned}
\left(\hat{\Psi}_{\alpha_{3}}^{3 \alpha_{2} \alpha_{1}}, \hat{\Psi}_{\alpha_{3}^{\prime}}^{3 \alpha_{2}^{\prime} \alpha_{1}^{\prime}}\right) & =\sum_{r_{3} r_{3}^{\prime}}\left(v_{\alpha_{3} r_{3}}^{3 \alpha_{2} \alpha_{1}}, v_{\alpha_{3}^{\prime} r_{3}^{\prime}}^{3 \alpha_{2}^{\prime} \alpha_{1}^{\prime}}\right)^{s}\left(z_{\alpha_{3} r_{3}}^{3 \alpha_{2} \alpha_{1}}, z_{\alpha_{3}^{\prime} r_{3}^{\prime}}^{3 \alpha_{2}^{\prime} \alpha_{1}^{\prime}}\right)^{e} \\
& \propto \delta_{\alpha_{3}^{\prime} \alpha_{3}} \delta^{\alpha_{2}^{\prime} \alpha_{2}} \delta^{\alpha_{1}^{\prime} \alpha_{1}} .
\end{aligned}
$$

The idea of strong decoherence is that we require orthogonality of the $z$ in the past alternatives, viz.,

$$
\left(z_{\alpha_{3} r_{3}}^{3 \alpha_{2} \alpha_{1}}, z_{\alpha_{3}^{\prime} r_{3}^{\prime}}^{3 \alpha_{1}^{\prime} \alpha_{1}^{\prime}}\right)^{3 e} \propto \delta^{\alpha_{2} \alpha_{2}^{\prime}} \delta^{\alpha_{1} \alpha_{1}^{\prime}}
$$

for all $\alpha_{3}, \alpha_{3}^{\prime}, r_{3}$, and $r_{3}^{\prime}$. Note that we do not require orthogonality in the index $\alpha_{3}$. There is no need for it. Orthogonality in $\alpha_{3}$ is automatic from (6.3) once (6.6) is satisfied. Further, as we will see in the next section, the $z$ are connected with records of the histories and physically it takes some time for records to form. Nonorthogonality in $\alpha_{3}$ is consistent with that.

It is easy to see that strong decoherence is a stronger condition than medium decoherence. Strong decoherence ensures that

$$
\left(\hat{\Psi}_{\alpha_{3}}^{3 \alpha_{2} \alpha_{1}}, \hat{\mathcal{O}} \hat{\Psi}_{\alpha_{3}^{\prime}}^{3 \alpha_{2}^{\prime} \alpha_{1}^{\prime}}\right) \propto \delta^{\alpha_{2} \alpha_{2}^{\prime}} \delta^{\alpha_{1}^{\prime} \alpha_{1}^{\prime}}
$$

for any operator $\hat{\mathcal{O}}$ of the form

$$
\hat{\mathcal{O}}^{3 s}=\hat{\mathcal{O}}^{3 s} \otimes I^{3 e},
$$

not just for $\hat{\mathcal{O}}^{3 s}=I^{3 s} \otimes I^{3 e}=I$, which is all that medium decoherence ensures.

As defined here, strong decoherence requires only that the projections defining the branches commute at each time (5.6). There is a system-environment split for each time, but the split generally changes from one time to the next. When the set of histories is a narrative all the projections at different times commute (4.1). There is then a common framework for all projections at all times (5.7) and correspondingly a notion of a system-environment split for all times. The notion of system, that which is followed in common by all the projections, will necessarily be more restricted than it is at one time unless the projections at different times are connected by a very simple narrative rule (e.g., identity). That is because there will be many more intersections to consider in the construction of the common framework as in Sec. VD. That does not make strong decoherence easier, but it would enable system and environment to be followed separately over time, as will be described in the next section.

We now turn to describing some consequences of this strong decoherence condition.

\section{CONSEQUENCES OF STRONG DECOHERENCE}

\section{A. Records}

A record of a history is an alternative at one time that has a high probability of correlation with alternatives in the history at an earlier time. A set of alternative histories is said to be recorded if there is a set of alternatives at some time, one of which is correlated with each past history in the set. To see how this idea is implemented in the present framework we continue with just two time steps represented by class operators

$$
\hat{C}_{\alpha_{2} \alpha_{1}}^{2}=\hat{P}_{\alpha_{2}}^{2 \alpha_{1}} e^{-i H\left(t_{2}-t_{1}\right) / \hbar} \hat{P}_{\alpha_{1}}^{1} e^{-i H\left(t_{1}-t_{0}\right) / \hbar} .
$$


The illustration with this simple case should be sufficient to see how to generate more general formulas with more steps.

This set of histories represented by (7.1) is recorded at time step $t_{3}$ if there is a set of commuting, orthogonal projections $\left\{\hat{R}_{\alpha_{2} \alpha_{1}}^{3}\right\}$ satisfying [cf. (2.1)]

$$
\hat{R}_{\alpha_{2} \alpha_{1}}^{3} \hat{R}_{\alpha_{2}^{\prime} \alpha_{1}^{\prime}}^{3}=\delta_{\alpha_{1} \alpha_{1}^{\prime}} \delta_{\alpha_{2} \alpha_{2}^{\prime}} \hat{R}_{\alpha_{2} \alpha_{1}}^{3}
$$

such that

$$
\hat{R}_{\alpha_{2} \alpha_{1}}^{3} \hat{\Psi}^{3}=\hat{\Psi}_{\alpha_{2}}^{3 \alpha_{1}} \equiv e^{-i H\left(t_{3}-t_{2}\right) / \hbar} \hat{C}_{\alpha_{2} \alpha_{1}}^{2} \hat{\Psi}^{0} .
$$

Taking a time step after the last one in the histories captures the idea that it might take time for a record to form.

As a consequence of strong decoherence there are always records of past history in the environment satisfying (7.2). Examples can be exhibited explicitly. In the environment Hilbert space $\mathcal{H}^{3 e}$ at time step 3 define the following set of projections:

$$
\hat{R}_{\alpha_{2} \alpha_{1}}^{3 e}=\operatorname{Proj}\left(z_{\alpha_{3} r_{3}}^{3 \alpha_{2} \alpha_{1}}\right)
$$

and

$$
\hat{R}_{\alpha_{2} \alpha_{1}}^{3} \equiv I^{s} \otimes \hat{R}_{\alpha_{2} \alpha_{1}}^{3 e} .
$$

Here Proj means projections on the subspace of $\mathcal{H}^{3 e}$ spanned by $z_{\alpha_{3} r_{3}}^{3 \alpha_{2} \alpha_{1}}$ as $\alpha_{3}$ and $r_{3}$ vary. It is then a straightforward calculation using the strong decoherence condition (6.6) to verify that with these definitions the record conditions (7.2) are satisfied. Since the $z$ are generally not a basis in the environment Hilbert space, there will generally be other choices of record operators satisfying (7.2) containing those in (7.3).

Note that were the analogous construction of the $R$ made at $t_{2}$, the last time of the history, it would not have worked. Mathematically that is because the strong decoherence condition on the $z$ at that time would not have ensured orthogonality in $\alpha_{2}$. That is consistent with the physical idea that records of alternatives are not available instantaneously but generally take some time to form.

Many mechanisms of decoherence that have been studied in simple models involve a coupling of a followed system to an environment of different degrees of freedom. The environmental degrees of freedom carry away the phases between alternative histories of the followed degrees of freedom and produce decoherence. After the interaction the environmental degrees of freedom contain records of the configuration of the followed system at the time of interaction. ${ }^{15}$ Both environments and records in environments are consequences of strong decoherence, as we have seen in this section and in Sec. V. Restricting quasiclassical realms to strongly decoherent histories of quasiclassical variables thus captures, in a general way, key features of realistic mechanisms of decoherence.

\section{B. Permanence of the past}

We experience the present, remember the past, and try to predict the future. We have the impression that the future is

\footnotetext{
${ }^{15}$ See, e.g., [31] for records in the oscillator models. For an emphasis on the redundancy of records see, e.g., [32].
}

uncertain, waiting to happen. By contrast, the past is over, done with, and permanent even if our knowledge of what happened is uncertain. However, these subjective ways of organizing temporal information and these impressions are not built into the fundamental laws of the quantum universe. Plausibly they rather arise from our particular construction as physical systems within the universe ${ }^{16}$ [33]. At every moment of time in our history of there is a present separating a past from a future.

Consider the present, past, and future at a particular moment in our history. In decoherent histories quantum theory there is no essential difference between using present data to predict the future and using it to retrodict the past [2,34]. Both prediction and retrodiction involve the probabilities of histories conditioned on present data, one of histories that extend toward the big bang (the past) and the other away from it (the future). ${ }^{17}$ One gives probabilities of what did happen, the other of what will happen.

There may be many realms extending the present towards the future and many towards the past. Neither past or future is therefore unique [34]. However, usually we are concerned with the past or future of a quasiclassical realm. We will assume that here.

Extending a quasiclassical realm into the future risks losing the ability to retrodict the past. That is because any extension into the future is a fine graining of the present set of alternative histories. A coarse graining of a decoherent set of alternative histories is decoherent. A fine graining may not be. Extending a realm to the future risks losing the decoherence of the past. The past is therefore not necessarily permanent (see, e.g., [34]).

Strong decoherence ensures the permanence of the past. That is because the condition (6.6) requires the decoherence of past alternatives. A more physical way of saying this is that, as discussed above, strong decoherence ensures the existence of present records for the past that ensure its decoherence and permanence.

\section{Density matrices}

When there is a system-environment split of the Hilbert space at any one time $k$ as in (6.1) it is possible to define a system density matrix $\rho^{k s}$ by tracing over the environment. Specifically,

$$
\rho^{k s}=\operatorname{Sp}\left(\hat{\Psi}^{k} \hat{\Psi}^{k^{\dagger}}\right),
$$

where $\mathrm{Sp}$ means the trace over the environment Hilbert space $\mathcal{H}^{k e}$. The expected value of any system observable of the form (6.8) at time step $k$ can be calculated just from $\rho^{k s}$, viz.,

$$
\langle\hat{\mathcal{O}}\rangle^{k} \equiv \operatorname{Tr}\left(\hat{\mathcal{O}} \hat{\Psi}^{k} \hat{\Psi}^{k \dagger}\right)=\operatorname{tr}\left(\hat{\mathcal{O}}^{s} \rho^{k s}\right),
$$

where $\operatorname{Tr}$ is the trace over all of Hilbert space $\mathcal{H}$ and $\operatorname{tr}$ is the trace over the system part $\mathcal{H}^{k s}$.

In this section we show that strong decoherence implies this result on a branch by branch basis. Specifically we show the following. Define at time step $k$, for each branch $\alpha_{k-1} \cdots \alpha_{1}$,

\footnotetext{
${ }^{16}$ That is, an IGUS - an information gathering and utilizing system [7]

${ }^{17}$ However, the formulas for these probabilities differ in form [2].
} 
its branch density matrix in $\mathcal{H}^{k s}$,

$$
\rho^{k \alpha_{k-1} \cdots \alpha_{1} s} \equiv \operatorname{Sp}\left(\hat{\Psi}^{k \alpha_{k-1} \cdots \alpha_{1}} \hat{\Psi}^{k \alpha_{k-1} \cdots \alpha_{1} \dagger}\right) .
$$

Then if $\hat{\mathcal{O}}$ is a system observable of the form (6.8) for the system-environment split at time step $k$, strong decoherence implies

$$
\begin{aligned}
\langle\hat{\mathcal{O}}\rangle^{k} & \equiv \operatorname{Tr}\left(\hat{\mathcal{O}} \hat{\Psi}^{k} \hat{\Psi}^{k \dagger}\right)=\operatorname{tr}\left(\hat{\mathcal{O}}^{s} \rho^{k s}\right) \\
& =\sum_{\alpha_{k-1} \cdots \alpha_{1}} \operatorname{tr}\left(\hat{\mathcal{O}}^{s} \rho^{k \alpha_{k-1} \cdots \alpha_{1} s}\right) .
\end{aligned}
$$

We illustrate the demonstration with just three time steps as in Secs. III D and VI. The generalization to more steps should be straightforward. We begin by using (6.4) to write the expected value of a system observable (6.8) at time step 3 in terms of the $v$ and $z$,

$$
\begin{aligned}
\langle\hat{\mathcal{O}}\rangle^{3} & \equiv\left(\hat{\Psi}^{3}, \hat{\mathcal{O}}^{s} \hat{\Psi}^{3}\right) \\
& =\sum_{\alpha_{3} \alpha_{2} \alpha_{1}} \sum_{\alpha_{3}^{\prime} \alpha_{2}^{\prime} \alpha_{1}^{\prime}}\left(v_{\alpha_{3} r_{3}}^{3 \alpha_{2} \alpha_{1}},{ }^{s} \hat{\mathcal{O}}^{s} v_{\alpha_{3}^{\prime} r_{3}^{\prime}}^{3 \alpha_{2}^{\prime} \alpha_{1}^{\prime}}\right)^{s}\left(z_{\alpha_{3} r_{3}}^{3 \alpha_{2} \alpha_{1}}, z_{\alpha_{3}^{\prime} r_{3}^{\prime}}^{3 \alpha_{2}^{\prime} \alpha_{1}^{\prime}}\right)^{e} \\
& =\sum_{\alpha_{2} \alpha_{1}} \sum_{\alpha_{3} \alpha_{3}^{\prime}}\left(v_{\alpha_{3} r_{3}}^{3 \alpha_{2} \alpha_{1}}, s \hat{\mathcal{O}}^{s} v_{\alpha_{3}^{\prime} r_{3}^{\prime}}^{3 \alpha_{2} \alpha_{1}}\right)^{s}\left(z_{\alpha_{3} r_{3}}^{3 \alpha_{2} \alpha_{1}}, z_{\alpha_{3}^{\prime} r_{3}^{\prime}}^{3 \alpha_{2} \alpha_{1}}\right)^{e} .
\end{aligned}
$$

The last equality is a consequence of the strong decoherence condition (6.6). The result gives the expected value of a system observable $\langle\hat{\mathcal{O}}\rangle^{3}$ as a single sum over branches $\alpha_{2}, \alpha_{1}$. To put it differently, strong decoherence means that there is no interference between branches. Expanding (7.6) in a similar way in terms of the $v$ and $z$ shows that the matrix elements of $\rho^{k \alpha_{k-1} \cdots \alpha_{1} s}$ in the basis of the $\left\{v_{\alpha_{3} r_{3}}^{3 \alpha_{2} \alpha_{1}}\right\}$ in the system Hilbert space $\mathcal{H}^{3 s}$ are $\left(z_{\alpha_{3} r_{3}}^{3 \alpha_{2} \alpha_{1}}, z_{\alpha_{3}^{\prime} r_{3}^{\prime}}^{3 \alpha_{2} \alpha_{1}}\right)^{e}$. Equation (7.8) is then (7.7) in this particular basis.

We note that diagonality of the density matrices $\rho^{k \alpha_{k-1} \cdots \alpha_{1} s}$ is not a consequence of strong or medium decoherence. Diagonality would mean that

$$
\left(z_{\alpha_{3} r_{3}}^{3 \alpha_{2} \alpha_{1}}, z_{\alpha_{3}^{\prime} r_{3}^{\prime}}^{3 \alpha_{2}^{\prime} \alpha_{1}^{\prime}}\right)^{3 e} \propto \delta_{\alpha_{3} \alpha_{3}^{\prime}} \delta^{\alpha_{2} \alpha_{2}^{\prime}} \delta^{\alpha_{1} \alpha_{1}^{\prime}},
$$

which is a stronger condition than strong decoherence. ${ }^{18}$ This condition is not necessary for medium decoherence. The medium decoherence condition (3.9) is satisfied in $\alpha_{3}$ automatically as a consequence of the orthogonality of the $v$ as (6.5) shows. Further, strong decoherence is equivalent to the creation of records in the environment as we showed

\footnotetext{
${ }^{18}$ The condition is so strong that it would imply that the expansion (7.8) is a Schmidt decomposition, which would fix the variables in the projections and risk conflict with our assumption that they are quasiclassical.
}

in Sec. VII A. One would expect that physical records would not appear simultaneously with the alternative, but take some time to form. Models of decoherence may lead to a density matrix becoming diagonal after time, ${ }^{19}$ but that plays no role in the fundamental formulation of decoherent histories quantum theory.

\section{CONCLUSION}

If the universe is indeed a quantum mechanical system then at a fundamental level the predictions of theory are the probabilities of the individual members of sets of alternative coarse-grained histories: realms. Of particular interest are the quasiclassical realms that are a feature of our universe, extending over the whole of its visible part from just after the big bang to the far future. These describe almost everything we observe from every day scales to those of cosmology. Characterizing the universe's quasiclassical realms is therefore an important problem in quantum mechanics.

This paper has continued a program of characterizing the quasiclassical realms in decoherent histories quantum theory when quantum gravity is neglected and classical space-time is assumed. This paper discussed the ideas of adaptive branchdependent coarse grainings, narrative sets of histories, and strong decoherence. Putting together all the elements of this paper and our previous ones, we can characterize a quasiclassical realm as a strongly decoherent set of alternative histories, defined by an adaptive branch-dependent coarse graining built on a narrative framework of quasiclassical variables, exhibiting with high probability patterns of correlation in time described by closed sets of deterministic equations, and maximally refined consistent with all these properties.

\section{ACKNOWLEDGMENTS}

We thank the Aspen Center for Physics for hospitality over several summers while this work was in progress. J.B.H. thanks the Santa Fe Institute for supporting many visits there. The work of J.B.H. was supported in part by the National Science Foundation under Grants No. PHY05-55669, No. PHY08-55415, and No. PHY12-05500. The work of M.G.-M. was supported by the COUQ Foundation, by Insight Venture Management, and by the KITP in Santa Barbara. The generous help provided by these organizations is gratefully acknowledged.

\footnotetext{
${ }^{19}$ This occurs, for example, in some oscillator models; see, e.g., [4], Sec. II.6.4.
}

[1] J. B. Hartle, Found. Phys. 41, 982 (2011). A slightly shorter version is published in Many Quantum Worlds, edited by A. Kent and S. Saunders (Oxford University Press, Oxford, 2009).

[2] M. Gell-Mann and J. B. Hartle, in Complexity, Entropy, and the Physics of Information, edited by W. Zurek, SFI Studies in the Sciences of Complexity Vol. VIII (Addison-Wesley, Reading, MA, 1990).
[3] M. Gell-Mann and J. B. Hartle, in Proceedings of the 25th International Conference on High Energy Physics, Singapore, 1990, edited by K. K. Phua and Y. Yamaguchi (World Scientific, Singapore, 1990).

[4] J. B. Hartle, in Quantum Cosmology and Baby Universes, edited by S. Coleman, J. B. Hartle, T. Piran, and S. Weinberg, Proceedings of the 1989 Jerusalem Winter School for Theoretical Physics (World Scientific, Singapore, 1991), pp. 65-157. 
[5] M. Gell-Mann and J. B. Hartle, Phys. Rev. D 47, 3345 (1993).

[6] M. Gell-Mann and J. B. Hartle, in The Physical Origins of Time Asymmetry, edited by J. Halliwell, J. Pérez-Mercader, and W. Zurek (Cambridge University Press, Cambridge, 1994); J. B. Hartle, Phys. Rev. D 49, 6543 (1994).

[7] M. Gell-Mann, The Quark and the Jaguar (Freeman, New York, 1994).

[8] M. Gell-Mann and J. B. Hartle, arXiv:gr-qc/9404013.

[9] J. B. Hartle, in Proceedings of the Cornelius Lanczos International Centenary Conference, edited by J. D. Brown, M. T. Chu, D. C. Ellison, and R. J. Plemmons (SIAM, Philadelphia, 1994).

[10] M. Gell-Mann and J. B. Hartle, in Proceedings of the Fourth Drexel Symposium on Quantum Non-Integrability-The Quantum-Classical Correspondence, edited by D.-H. Feng and B.-L. Hu (International Press, Boston, 1995).

[11] M. Gell-Mann and J. B. Hartle, Phys. Rev. A 76, 022104 (2007).

[12] R. B. Griffiths, Consistent Quantum Theory (Cambridge University Press, Cambridge, 2002).

[13] R. Omnès, Interpretation of Quantum Mechanics (Princeton University Press, Princeton, 1994).

[14] J. B. Hartle, in Directions in General Relativity, edited by B.-L. $\mathrm{Hu}$, M. P. Ryan, and C. V. Vishveshwara (Cambridge University Press, Cambridge, 1993), Vol. 1.

[15] W. Zurek, Rev. Mod. Phys. 75, 715 (2003).

[16] J. B. Hartle, in Quo Vadis Quantum Mechanics, edited by A. Elitzur, S. Dolev, and N. Kolenda (Springer, Heidelberg, 2005), pp. 73-82.

[17] J. B. Hartle, in Gravitation and Quantizations, edited by B. Julia and J. Zinn-Justin, Proceedings of the Les Houches
Summer School of Theoretical Physics, LVII (North-Holland, Amsterdam, 1995).

[18] J. B. Hartle, in The Quantum Structure of Space and Time, Proceedings of the 23rd Solvay Conference on Physics, edited by D. Gross, M. Henneaux, and A. Sevrin (World Scientific, Singapore, 2007).

[19] For a small subset of the possible examples of this see, e.g., J. B. Hartle, Phys. Rev. D 44, 3173 (1991); D. Marolf, Class. Quantum Grav. 12, 2469 (1995); J. J. Halliwell, Phys. Rev. D 80, 124032 (2009).

[20] L. Diósi, Phys. Rev. Lett. 92, 170401 (2004).

[21] J. J. Halliwell, Phys. Rev. D 58, 105015 (1998).

[22] J. J. Halliwell, Phys. Rev. Lett. 83, 2481 (1999).

[23] M. Srednicki and J. B. Hartle, Phys. Rev. D 81, 123524 (2010); arXiv:1004.3816.

[24] J. B. Hartle (unpublished).

[25] U. Fano, Rev. Mod. Phys 29, 74 (1957).

[26] E. Joos and H. D. Zeh, Z. Phys. B 59, 223 (1985).

[27] R. P. Feynman and J. R. Vernon, Ann. Phys. (NY) 24, 118 (1963).

[28] A. Caldeira and A. Leggett, Physica A 121, 587 (1983).

[29] T. A. Brun and J. B. Hartle, Phys. Rev. D 60, 123503 (1999).

[30] J. Finkelstein, Phys. Rev. D 47, 5430 (1993).

[31] J. J. Halliwell, Phys. Rev. D 60, 105031 (1999).

[32] See, e.g., W. Zurek, Nat. Phys. 5, 181 (2009); C. J. Reidel and W. Zurek, arXiv:1312.0331.

[33] J. B. Hartle, Am. J. Phys. 73, 101 (2005).

[34] J. B. Hartle, Phys. Scr. T76, 67 (1998). 